\title{
The unexpected malleability of lignin
}

\author{
Curcumin, an aromatic diarylheptanoid, is a principal component of turmeric (Curcuma longa), commonly \\ used in Asian cooking, giving curry its orange colour. Introducing two enzymes into Arabidopsis thaliana caused \\ incorporation of curcumin into its lignin polymer, enhancing sugar release from the cell wall and turning it yellow.
}

\section{Gerald A. Tuskan}

ignin's origins are thought to coincide with the advent of the first land plants. The pathway leading to lignin formation favoured desiccation tolerance, ultraviolet protection and reproductive success. The lignin biosynthetic machinery was subsequently co-opted during the phylogenetic diversification of land plants, leading to the creation of specieslevel variation in cell anatomy and cell wall chemistry, which enabled variation of water movement within a plant and pathogen resistance. The shared chemistry and anatomy of plant cell walls led to the historical view that lignin varied only within the confines of three fundamental subunits - hydroxyphenyl $(\mathrm{H})$, guaiacyl $(\mathrm{G})$ and syringyl (S). Now, in a paper published in Nature Plants, Oyarce et al. ${ }^{1}$ report the incorporation of diferuloylmethane (curcumin) into lignin deposited in the cell walls of transgenic Arabidopsis thaliana. As a result, sugar release (or saccharification) efficiency was increased by $15-22 \%$ in the transgenic lines, which corresponds to enhanced biorefining efficiencies.

The classical view of lignin biosynthesis regulation and composition are rapidly changing. We have known for some time that lignin is composed of three monomers - p-coumaryl, coniferyl and sinapyl alcohol; from the $\mathrm{H}, \mathrm{G}$ and $\mathrm{S}$ subunits, respectively. $\mathrm{H} / \mathrm{G} / \mathrm{S}$ ratios vary among genotypes, and between transgenic and wild-type plants. Reductions in lignin content facilitate the increase in industrial value (valorization) of plant biomass ${ }^{2}$. Recently it has been shown that the regulation of monomer formation occurs upstream of the phenylalanine pathway ${ }^{3}$, that there are alternate pathways to final lignin biosynthesis ${ }^{4}$ and that mature lignin can contain alternate aromatic units ${ }^{5}$. Oyarce et al. ${ }^{1}$ have now demonstrated that a molecule not known to be a standard component of the lignin polymer, curcumin, can be biosynthesized and incorporated into the lignin polymer.

Curcumin, the molecule that gives turmeric its orange hue, is not known to be a normal component of lignin. As well as turning the cell walls of the transgenic plants yellow, the incorporation of curcumin as an ester into lignin allowed facile depolymerization following treatment with mild alkali. The new biomass is more easily converted to monosaccharide sugars in saccharification experiments and, although the improvements are modest, this proof-of-principle implies that other non-native aromatic compounds could be incorporated into lignin, which will have more substantial effects.

Previous studies have modified the H/G/S ratios of lignin ${ }^{7}$, incorporated pathway intermediates such as cinnamaldehydes, ferulic acid and 5-hydroxyconiferyl alcohol into the polymer ${ }^{6}$, and have shown that alternate monolignol monomers outside the standard synthesis pathway (for example, tricin, isoraponthigenin and resveratrol) can be present in lignin ${ }^{8-10}$. The results presented by Oyarce et al. ${ }^{1}$ expand the suite of aromatic compounds that have been cross-linked into the lignin polymer. The unexpected flexibility in lignin composition unlocks the possibility that it can may be tailored to suit industrial or environmental purposes. With this knowledge, and the application of new approaches such as CRISPR, it is possible to realize the goal of several researchers of incorporating new subunits into the lignin polymer to increase the efficiency of lignin breakdown during biomass processing ${ }^{11}$.

There are several remaining questions related to future applications of this discovery. The constitutive expression of DIKETIDE-COA SYNTHASE and CURCUMIN SYNTHASE 2 resulted in the formation of many additional compounds beyond curcumin, mostly phenylpentanoids. Could additional carbon be diverted to curcumin biosynthesis if a more specific CURCUMIN SYNTHASE is found? Will similar improvements be seen in biomass crops such as poplar, miscanthus or switchgrass? Field testing of the transgenic plants was not performed, and so questions remain over how such plants will succeed when grown in the field. Does curcumin incorporation into the cell wall affect drought tolerance, nutrient uptake or pest resistance? Most important is the question of whether the introduction of new molecules into lignins can be used to chemically enable simpler depolymerization or the production of new materials from the lignin polymer, as either could greatly increase the routes for valorization of lignin polymers.

Gerald A. Tuskan DOE Center for Bioenergy Innovation in the Biosciences Division of Oak Ridge National Laboratory, Oak Ridge, TN, USA. e-mail:gtk@ornl.gov

Published online: 28 January 2019 https://doi.org/10.1038/s41477-019-0360-9

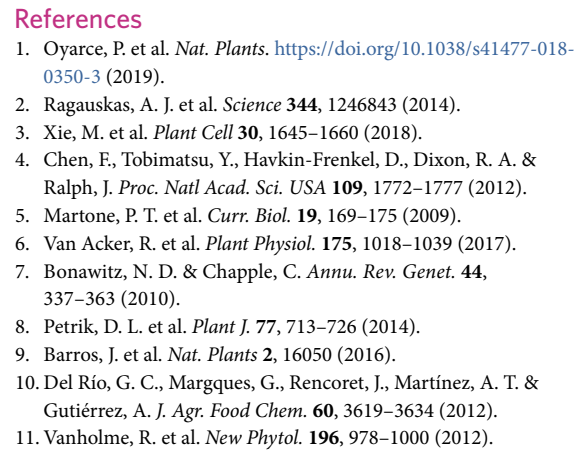

The author declares no competing interests. 